\title{
Cyst of the Nuck Canal: A Rare Cause of Painful Inguinal Swelling in Women Who Are Not To Be Misunderstal
}

\author{
Mohamed Ait Chtouk ${ }^{1 *}$, Labretesche Gracia Christ Gakosso ${ }^{1}$, Soumia Alj ${ }^{1}$
}

${ }^{1}$ Radiology Department, CHU Mohammed VI, Marrakech, Cadi Ayyad University, Marrakesh, Morocco

DOI: $10.36347 /$ simcr.2021.v09i04.039

| Received: 18.03.2021 | Accepted: 26.04.2021 | Published: 29.04.2021

*Corresponding author: Ait Chtouk Mohamed

Abstract

The cyst of the Nuck canal often called female hydrocele is a rather rare pathology due to the persistence of the peritoneo-vaginal canal in women. Frequently described and observed in pediatric settings, its revelation in adulthood is very rare and has been very much described in the literature. We report here the case of a 20 -year-old patient, with a history of right inguinal herniated cure not strangled in childhood, with the notion of appendectomy 07 months ago. The reason for consultation was a hyperalgic and disabling right inguinfaction evolving in an apyretic context without transit disorders. Emergency abdominal ultrasound revealed a cystic formation in relation to the right inguinal ligament, oblong in shape, with finely echogenic contents, fine wall, no partition, no vegetation, or endokystic intestinal content. The exploration was supplemented by an abdominal CT scan. This observation reveals the place of imaging in the differential diagnosis of painful pelvic swelling in women.

Keywords: Cyst, Nuck canal, imagery.

Copyright $\odot 2021$ The Author(s): This is an open-access article distributed under the terms of the Creative Commons Attribution 4.0 International License (CC BY-NC 4.0) which permits unrestricted use, distribution, and reproduction in any medium for non-commercial use provided the original author and source are credited.

\section{INTRODUCTION}

The Kyste of the Nuck Canal was first described by the Dutch anatomist Anton Nuck in 1691; often called female hydrocele in women, it is a rather rare complication due to the persistence of the peritoneo-vaginal canal in women $[1,2]$.

It was first frequently described and observed in paediatric settings with a prevalence in the range of 1 to $4 \%$ [3]. Its revelation in adulthood is very rare and has been very much described in the literature [2, 3]. We report the case of a 20 -year-old patient, our goal is to illustrate the contribution of imaging in the positive diagnosis of this pathological entity quite rare in adults.

\section{ObServation}

A 20-year-old patient, nulligeste and nullipare, admitted to the emergency room for a right inguinal pain, intermittently evolving for 03 months, started with an discomfort in the groin, then gradually became painful, accompanied by a slight swelling of the right groin. The patient reports a history of right inguinal hernia cure in childhood and an appendectomy about seven (7) months ago.

The physical examination, objectived a slight swelling of the right groin, accompanied by intense pain at the palpation of the right inguinal fold radiating to the right iliac pit, with a slight defense of it, motivating the realization of an ultrasound of the inguinal region.

Ultrasound of the inguinal region was done using a superficial probe showing the presence of a juxtavascular, thin-walled, oblong-shaped, finely echogenic, partition-free, vegetation-free, detectable parietal defect or continuity with digestive handles (Figure-1). The unavailability of MRI emergency, led to further exploration by pelvic CT (CT) with and without injection of contrast product. CT imaging of a cystic formation of the right inguinal canal without continuity with digestive or gynecological structures with discrete infiltration of neighbourhood fat in relation to a reworked nueck canal hydrocele or cyst (Figure-2). 

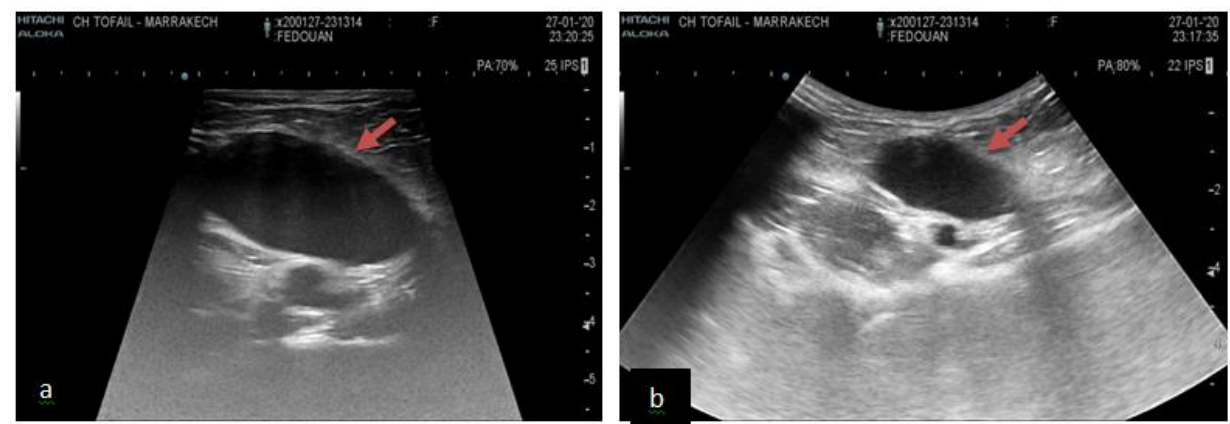

Fig-1: Longitudinal (a) and transverse (b) cut using a superficial probe, next to the right inguinal folds highlights a right femoral juxtavascular cystic formation (arrow), fine-walled, oblong-shaped, finely echogenic content, no partition, no vegetation, no detectable parietal defect or continuity with the digestive handles
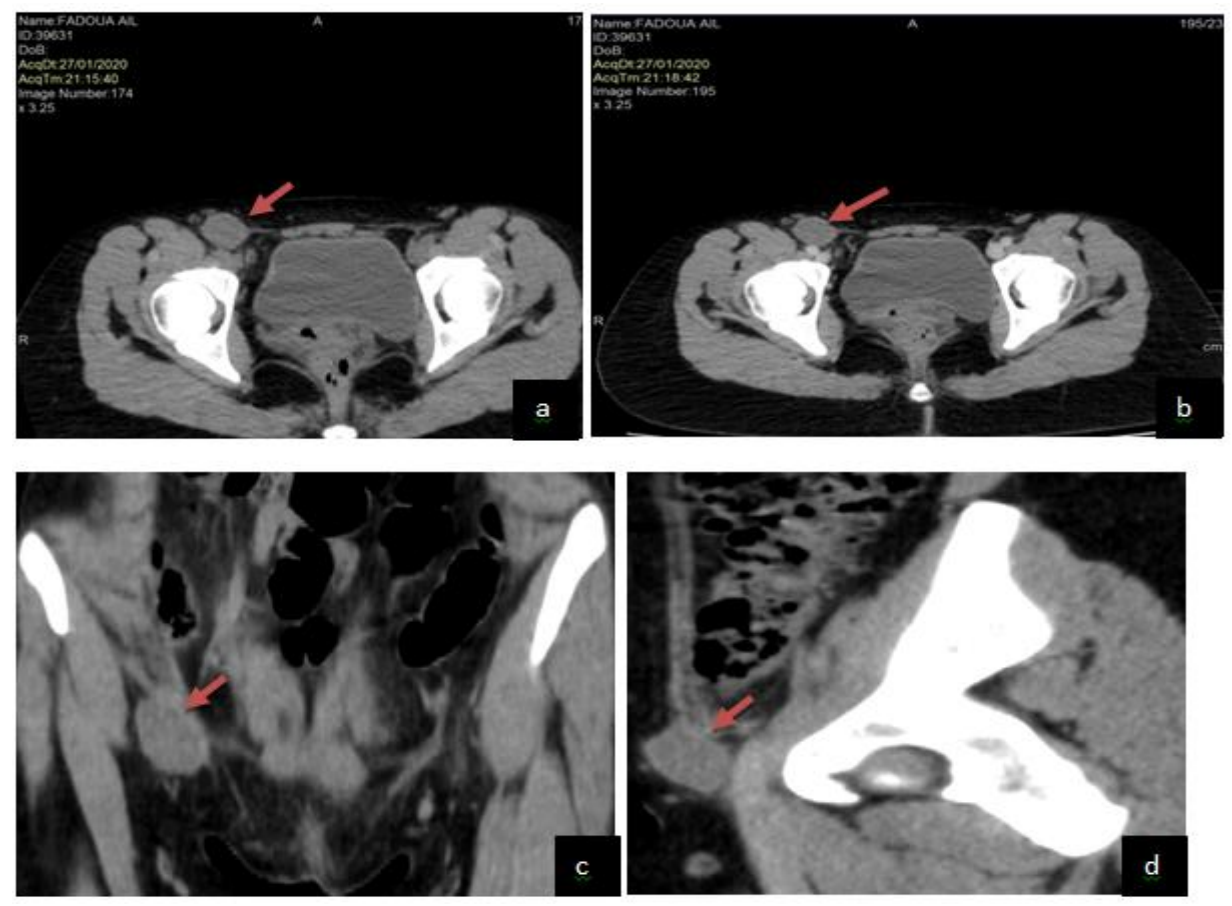

Fig-2: Abdomino-pelvic CT scan without (a, b, and c) and with injection (b) of contrast product, finds a common right femoral kystata-vascular formation (arrow), thin-walled, oblong-shaped, hypodense content, no partition, no vegetation, no detectable parietal defect or continuity with the digestive handles responsible for a reduction in caliber of the right common femoral vein that remains permeable

\section{DiscUSSION}

The cyst of the Nuck canal is a very rare nosological entity due to a defect in the closure of the distal part of the peritoneo-vaginal canal that becomes enkyst and usually manifests itself in painless swelling in relation to the inguinal ligament and/or labium in adolescents. When symptomatic, it can be revealed by dull or intense pain in the groin; as was the case with our patient [1-4].

In some situations a hernia of the bladder, ovaries and fallopian tubes can be observed in the Nuck Canal which is exceptional [3].

Imaging through these different techniques occupies a prominent place in the pre-operative diagnosis of this pathology. Less expensive, nonirradiating and highly accessible ultrasound provides irrefutable diagnostic elements, showing a very limited anechogenic, rounded or oval-shaped cystic formation, with a thin, uni or multi-layered non-vascularized doppler color, superficially and mediated to the pubic bones in the inguinal canal generating in some cases a posterior reinforcement. It can be the seat of endokystic partitions and or fine echoes [3-5] as in our case.

In addition to ultrasound, cross-sectional imaging can be performed to better study the reports of the lesion without substituting for ultrasound, which remains the first-line imaging examination. For this purpose, magnetic resonance imaging (MRI) represents the standard gold when available and feasible, on the other hand; it could not be performed in our patient due to the unavailability of MRI in our work environment [5].

However, it should be noted that the MRI results in the hydrocele or cyst of the Nuck. Canal have 
been described only in very few cases in the literature, manifesting as a cystic lesion in relation to the inguinal region, although limited, thin-walled, oval-shaped, which is hyper intense in T2 and hypo intense in T1 [3, $5]$.

In addition, a pelvic CT scan with emergency contrast injection in our patient in addition to ultrasound, found the presence of a projected cystic formation at the right, coarsely oval, fine-walled inguinal canal, which measured 40 x $18 \mathrm{~mm}$, spontaneously hypodense and unmodified after injection of contrast product. Kim AS et al., [4], described the same CT-scan semiology in their case published in 2016.

Differential diagnosis of cysts in the Nuckest Canal is mainly done with: Bartholin cyst, lymphadenopathy, indirect inguinal hernia and malignant and benign tumors. The main complications of the cyst or hydrocele of the Nuck canal are dominated mainly by: infection, haemorrhage or large cyst with retroperitoneal extension [6].

The treatment of choice remains surgery. A kystectomy is performed by incision of the lower groin in relation to swelling. In some cases, a laparoscopic with closure of the Nok Canal may be considered [3-6]. Our patient benefited from a kystectomy under general anesthesia with good post-operative development.

\section{CONCLUSION}

The cyst of the Nuck canal is a rare entity and must necessarily be considered in the differential diagnosis of pathologies of the inguinal region in young female adults. Medical imaging through abdominal ultrasound, due to its accessibility and safety, makes it possible to make the diagnosis, in front of a cystic formation in relation to the inguinal fold, however if in doubt cutting imaging may be recommended.

\section{REFERENCES}

1. Nasser H, King M, Rosenberg HK, Rosen A, Wilck E, Simpson WL. Anatomy and pathology of the canal of Nuck. Clin Imaging. 2018;51:83-92.

2. Choi YM, Lee GM, Yi JB, Yoon KL, Shim KS, Bae CW, Choi SI, Kim HC. Two cases of female hydrocele of the canal of nuck. Korean journal of pediatrics. 2012 Apr;55(4):143-6.

3. Rees MA, Squires JE, Tadros S, Squires JH. Canal of Nuck hernia: A multimodality imaging review. Pediatr Radiol. 2017;47:893-8.

4. Kim K.S., Choi J.H., Kim H.M. Hydrocele of the canal of Nuck in a female adult. Archives of plastic surgery. 2016;43(5):476-478.

5. Lai I, Page A, Hamidinia F, Rahmani R. Cysts of the canal of Nuck: A rare sonographic diagnosis. J Clin Ultrasound. 2017;45:175-8.

6. Caviezel A, Montet X, Schwartz J, Egger JF, Iselin CE. Female hydrocele: The cyst of Nuck. Urol Int. 2009;82:242-5. 